\title{
Study on Dynamic Adsorption Law of Binary System and Ternary System
}

\author{
Taoping Chen ${ }^{a}$, Zuoan Zhang ${ }^{b}$ and Bin Zhao ${ }^{c}$
}

The Key Laboratory of Enhancing Oil and Gas Recovery Efficiency of Educational Ministry, Northeast

Petroleum University, Daqing, Heilongjiang, China, 163318

aactp010@163.com, b1261864469@qq.com, c244802970@qq.com

Keywords: dynamic adsorption, betaine, mahogany sulfonate, binary system, ternary system

\begin{abstract}
Using Daqing natural sand packed tubular model and natural core, the injection rate is $1 \mathrm{~m} / \mathrm{d}$ and the experiment temperature is $45^{\circ} \mathrm{C}$, studying binary system and ternary system interfacial tension changes of the produced liquid after betaine-polymer binary system and weak alkali-mahogany sulfonate-polymer dynamic absorb to the natural core and sand packed tubular model with oil or without oil by using TX-500 interfacial tensiometer. The results show that: The adsorption migration law of binary system and ternary system in the natural core is consistent with the natural sand packed tubular model, the surfactant front often shows the characteristics of piston propulsion. The interfacial tension of the natural sand packed tubular model without residual oil is relatively stable, and the interfacial tension of the natural sand packed tubular model with residual oil fluctuates up and down. In the a natural sand packed tubular models without oil and natural cores, the adsorption capacity of betaine binary system is 5.4 and 6.5 times than mahogany sulfonate ternary system respectively, in the a natural sand packed tubular models with oil, the adsorption capacity of betaine binary system is 1.3 times than mahogany sulfonate ternary system. Agreement interfacial tension of binary or ternary flooding system - crude oil in the reservoir is not all in the original ultra-low, but depend on the emulsification of ultra-low interfacial tension within a radius of injection well and synergy with other components can effectively improve the oil recovery.
\end{abstract}

\section{Introduction}

Chemical flooding system will be adsorpted in the migration of reservoir, the loss of active components, directly affect the oil displacement effect, and cause the waste of chemical flooding agent and increase the operation cost. Therefore, the displacement effect of chemical flooding system is closely related to its adsorption in reservoir. Many scholars have studied the adsorption law of binary system and ternary system flooding system in oil layer ${ }^{[1-7]}$. The literature [1] shows that the dynamic adsorption capacity of the surfactant in the natural core was $0.081 \mathrm{mg} / \mathrm{g}$ after the $0.6 \mathrm{PV}$ ORS41 ternary system was injected into the natural core without residual oil. If the natural core contained residual oil, the dynamic adsorption capacity of the surfactant in the natural core was $0.061 \mathrm{mg} / \mathrm{g}$. The literature [2] shows the effect of the polymer on the static adsorption capacity of the surfactant on the surface of the sand was studied. The results showed that the concentration of the mahogany sulfonate was $0.05 \% \sim 0.3 \%$, the oil sands were mixed with the solution at the mass ratio of 1:9, adding polymer, surfactant adsorption on the surface of the sand decreases from $4.82 \mathrm{mg} / \mathrm{g}$ to $3.55 \mathrm{mg} / \mathrm{g}$. The literature [3] shows the dynamic retention of surfactant in rock core is less than its static adsorption in oil sand when $0.45 \mathrm{PV}$ heavy benzene sulfonate was injected into at the rate of $0.3 \mathrm{ml} / \mathrm{min}$ in the class II reservoir rocks. The saturated adsorption capacity of surfactant is greatly affected by the nature of core, and the dynamic retention of class II oil layer cores is higher than that of class I oil layer cores. The literature [4] shows the effects of core wettability and residual oil saturation on the adsorption capacity were studied. The core is more lipophilic, the adsorption of surfactant in the binary system is greater; the residual oil saturation is higher, the adsorption of surfactant is higher.

At present, the adsorption amount of surfactant in binary system and ternary system is evaluated by static adsorption and it is an evaluation criteria. Study on the injection amount of flooding system about dynamic adsorption law of core is reasonable PV number, and its value is small, which is far 
from saturated adsorption. The dynamic adsorption of oil flooding system is mainly determined by the concentration of injection fluid and produced fluid, in the process of dynamic adsorption, the interfacial tension between flooding system and crude oil is poorly understood. Therefore, in order to study the interfacial tension of flooding system - crude oil and the saturated adsorption quantity in the process of dynamic adsorption, Binary system and ternary system interfacial tension changes after they through the $50 \mathrm{~cm}$ sand packed model and natural core was tested. And study the adsorption law system in sand packed model and natural cores.

\section{Experimental Study}

\subsection{The main instruments and equipment.}

HBCD70 core displacement device of high temperature and high pressure, TX-500 interfacial tensiometer, Rs150 rheometer, constant pressure and constant speed pump, vacuum pump, metering pump, JJ-1 electric mixer, BS210S electronic balance, centrifugal glass tube with scale.

\subsection{The experimental materials.}

Binary system: Betaine surfactant, at a concentration of $0.3 \%$; Polymer: Daqing refining \& chemical company's production, molecular weight is $(700 \sim 950) \times 10^{4}$, at a concentration of 1680 $\mathrm{mg} / \mathrm{L}$.

Ternary system: Daqing mahogany sulfonate, at a concentration of $0.3 \%$; Polymer: Daqing refining $\&$ chemical company's production, molecular weight is $(700 \sim 950) \times 10^{4}$, at a concentration of 2720 mg/L; Alkali: $\mathrm{Na}_{2} \mathrm{CO}_{3}$, at a concentration of $1.2 \%$.

Experimental oil: Daqing fourth factory simulation oil and the viscosity is $9 \mathrm{~m} \mathrm{~Pa} \cdot \mathrm{s}$; Water samples: Daqing fourth factory X2 test area reinjection water; Saturated water: simulating Daqing original formation water (salinity is $6778 \mathrm{mg} / \mathrm{L}$ ); Oil sands: Daqing oilfield natural oil sands, washing, drying, screening; Sand packed tubular model parameters are shown in Table 1, natural core model parameters are shown in Table 2.

Table 1. Physical parameters of the sand packed tubular model

\begin{tabular}{|c|c|c|c|c|c|c|c|}
\hline Number & $\begin{array}{c}\text { Length } \\
(\mathrm{cm})\end{array}$ & $\begin{array}{c}\text { Diameter } \\
(\mathrm{cm})\end{array}$ & $\begin{array}{c}\text { Porosity } \\
(\%)\end{array}$ & $\begin{array}{c}\text { Air } \\
\text { permeability } \\
\left(10^{-3} \mu \mathrm{m}^{2}\right)\end{array}$ & $\begin{array}{c}\text { Water } \\
\text { permeability } \\
\left(10^{-3} \mu \mathrm{m}^{2}\right)\end{array}$ & $\begin{array}{c}\text { Saturation } \\
\text { of } \\
\text { irreducible } \\
\text { oil } \\
(\%)\end{array}$ & $\begin{array}{c}\text { Flooding } \\
\text { system }\end{array}$ \\
\hline 20160229 & 50 & 2.335 & 36.04 & 345.190 & 163.08 & 0 & binary \\
\hline 20160221 & 50 & 2.335 & 35.68 & 433.77 & 133.90 & 26.00 & binary \\
\hline $20151110-2$ & 50 & 2.335 & 36.94 & 358.26 & 128.97 & 0 & ternary \\
\hline $20151110-1$ & 50 & 2.335 & 35.68 & 445.0 & 164.72 & 25.67 & ternary \\
\hline
\end{tabular}

Table 2. Physical parameters of the natural core

\begin{tabular}{|c|c|c|c|c|c|c|}
\hline Number & $\begin{array}{c}\text { Length } \\
(\mathrm{cm})\end{array}$ & $\begin{array}{c}\text { Average } \\
\text { diameter } \\
(\mathrm{cm})\end{array}$ & $\begin{array}{c}\text { Porosity } \\
(\%)\end{array}$ & $\begin{array}{c}\text { Air } \\
\text { permeability } \\
\left(10^{-3} \mu \mathrm{m}^{2}\right)\end{array}$ & $\begin{array}{c}\text { Water } \\
\text { permeability } \\
\left(10^{-3} \mu \mathrm{m}^{2}\right)\end{array}$ & $\begin{array}{c}\text { Flooding } \\
\text { system }\end{array}$ \\
\hline $20170115^{(1)}$ & 49.915 & 2.52 & 29.26 & 596.43 & 409.4 & binary \\
\hline $20170518^{(2)}$ & 52.466 & 2.52 & 28.33 & 602.92 & 409.2 & ternary \\
\hline
\end{tabular}

Note: (1)20170115 cores are connected by 169-11,169-8,169-4,174-56,174-13,62-13.

(2) 20170518 cores are connected by 169-18,169-12,169-6,174-57,174-49,62-14.

\subsection{The experimental program.}

Taking into account the actual situation of oil in the reservoir, natural sand packed tubular models with oil or without oil and natural cores are used in the adsorption experiments. In order to facilitate the comparative study, the same experimental scheme was adopted for binary system and ternary system.

Dynamic adsorption model without oil : Using $50 \mathrm{~cm}$ sand packed tubular models and natural cores, air permeability measured, vacuum-pumping, and then saturated with water (calculated pore volume, porosity) and water permeability measured, and injecting binary system and ternary system; sampling 
the produced fluid at intervals of $0.1 \mathrm{PV}$, the interfacial tension was measured by the TX-500 interfacial tensiometer at $45^{\circ} \mathrm{C}$, while continuously recording produced liquid's interfacial tension until the value tends to the original ternary system's interfacial tension.

Dynamic adsorption model with oil: Using $50 \mathrm{~cm}$ sand packed tubular models, air permeability measured, vacuum-pumping, and then saturated with water (calculated pore volume, porosity) and water permeability measured, oil saturated and then aging, water flooding to water rate more than $98 \%$, and injecting binary system and ternary system; sampling the produced fluid at intervals of $0.1 \mathrm{PV}$, the interfacial tension was measured by the TX-500 interfacial tensiometer at $45^{\circ} \mathrm{C}$, while continuously recording produced liquid's interfacial tension until the value tends to the original ternary system's interfacial tension.

\section{The result and analysis of the experiment}

Sampling the produced fluid at intervals of $0.1 \mathrm{PV}$, the interfacial tension was measured by the TX-500 interfacial tensiometer, the dynamic adsorption law of binary or ternary flooding system was analyzed by using the relationship curve of interfacial tension and injection PV.

\subsection{The binary system adsorption law in the sand packed tubular models}

In order to analyze the influence of crude oil on adsorption law, two types of adsorption experiments were carried out by using sand packed tubular models with oil or without oil.

3.1.1 The binary system adsorption law in the sand packed tubular models without oil

Using the interfacial tension data, making the curve of the produced liquid's interfacial tension of sand packed tubular model (length $50 \mathrm{~cm}$ ) without oil and the binary system injection PV, it is shown in Fig. 1.

As can be seen from Fig. 1, the produced liquid of $50 \mathrm{~cm}$ in length sand packed tubular model 's interfacial tension changes relatively stable when the binary system injection PV is $0 \sim 16.0 \mathrm{PV}$, and the value is relatively high, all of the betaine was adsorbed on the surface of the oil sands approximately, after the number of injection PV is 16.0 PV, the interfacial tension decreases slowly, the betaine begins to flow out, the capacity of adsorption continues to increase, when the number of injection is $28.9 \mathrm{PV}$, and the interfacial tension reaches the state of original ultra-low, and with increase of the number of injection volume, the interfacial tension is stable and invariant, it can be considered when the injection volume of betaine is $28.9 \mathrm{PV}$, the amount of adsorption reaches the maximum, the saturated adsorption capacity of the oil sands is $18.92 \mathrm{mg} / \mathrm{g}$ by the calculation. Which indicate that the surfactant front is a non-piston type movement.

3.1.2 The binary system adsorption law in the sand packed tubular models with oil

Using the experimental data, making the curve of the produced liquid's interfacial tension of sand packed tubular model (length $50 \mathrm{~cm}$ ) with oil and the binary system injection PV, it is shown in Fig. 2.

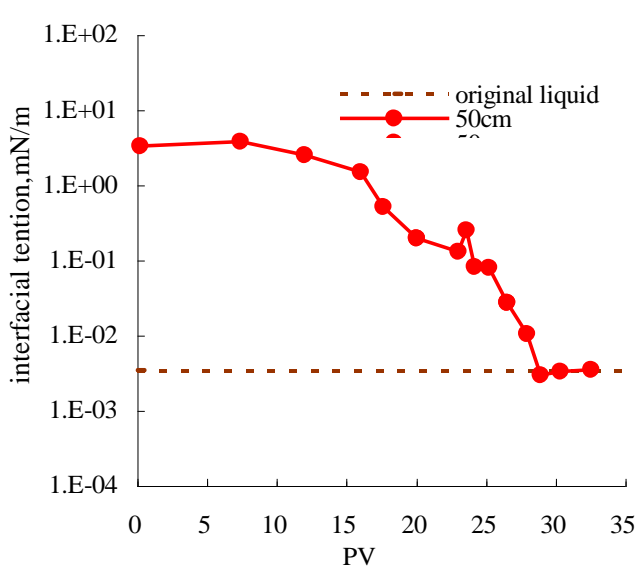

Fig.1 Relation of interfacial tension with the PV

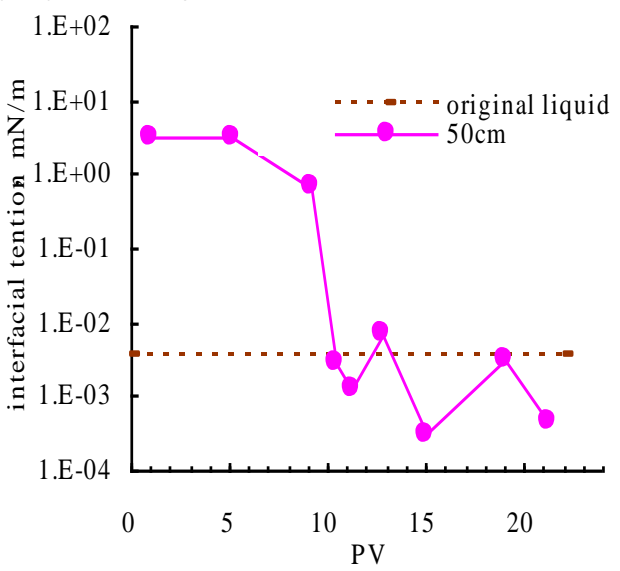

Fig.2 Relation of interfacial tension with the PV (with residual oil) 
As can be seen from Fig. 2, the produced liquid of $50 \mathrm{~cm}$ in length sand packed tubular model 's interfacial tension changes relatively stable when the binary system injection PV is $0 \sim 9.2 \mathrm{PV}$, and the value is relatively high, all of the betaine was adsorbed on the surface of the oil sands approximately, after the number of injection PV is 9.4 PV, the interfacial tension decreases rapidly, the betaine begins to flow out, the capacity of adsorption continues to increase, when the number of injection PV is 10.4, the interfacial tension reaches the state of ultra-low, compared with the natural sand packed tubular model's adsorption experiment without residual oil, with the increase of the amount of injection, the interfacial tension is not stable, but fluctuates within a certain range of ultra-low state, this is due to the oil changes in the produced liquid, which is causes the different content of the surfactant, it can be considered when the injection volume of betaine is $10.4 \mathrm{PV}$, the amount of adsorption reaches the maximum, the saturated adsorption capacity of this oil sand is $6.67 \mathrm{mg} / \mathrm{g}$ by the calculation. Which indicate that the surfactant front is a piston type movement.

\subsection{The ternary system adsorption law in the sand packed tubular models}

In order to compare with the adsorption law of binary system, two types of ternary system adsorption experiments were carried out by using sand packed tubular models with oil or without oil.

3.2.1 The ternary system adsorption law in the sand packed tubular models without oil.

Using the interfacial tension data, making the curve of the produced liquid's interfacial tension of sand packed tubular model (length $50 \mathrm{~cm}$ ) without oil and the ternary system injection PV, it is shown in Fig. 3.

As can be seen from Fig. 3, The produced liquid of $50 \mathrm{~cm}$ in length sand packed tubular model 's interfacial tension changes relatively stable when the ternary system injection PV is $0 \sim 5.1 \mathrm{PV}$, and the value is relatively high, all of the ternary was adsorbed on the surface of the oil sands approximately, when the number of injection PV is 5.1, the interfacial tension decreases rapidly, ternary begins to flow out, the capacity of adsorption continues to increase, when the number of injection is 5.3 PV, and the interfacial tension reaches the state of original ultra-low, and with increase of the number of injection volume, the interfacial tension is stable and invariant, it can be considered when the injection volume of betaine is $5.3 \mathrm{PV}$, the amount of adsorption reaches the maximum, the saturated adsorption capacity of this oil sands is $3.52 \mathrm{mg} / \mathrm{g}$ by the calculation. Which indicate that the surfactant front is a piston type movement.

3.2.1 The ternary system adsorption law in the sand packed tubular models with oil

Using the interfacial tension data, making the curve of the produced liquid's interfacial tension of sand packed tubular model (length $50 \mathrm{~cm}$ ) with oil and the ternary system injection PV, it is shown in Fig.4.

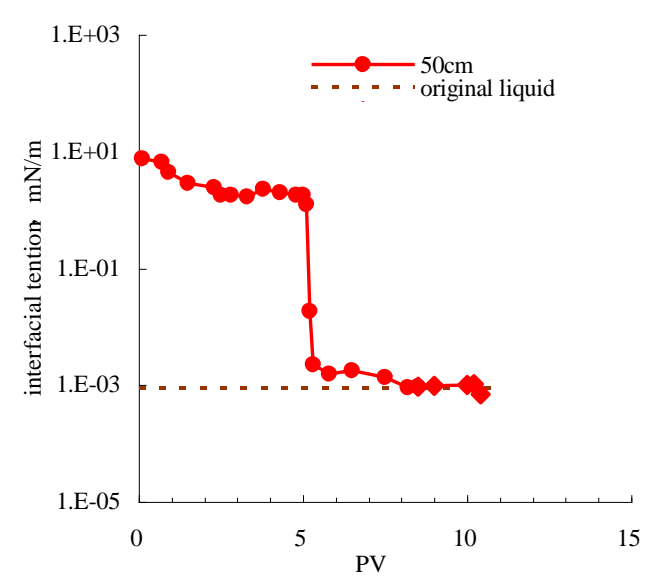

Fig.3 Relation of interfacial tension with PV

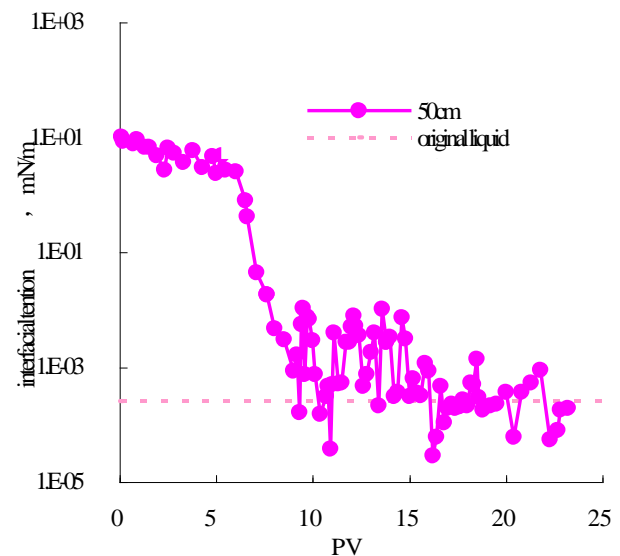

Fig.4 Relation of interfacial tension with PV (with residual oil)

As can be seen from Fig. 4, the produced liquid of $50 \mathrm{~cm}$ in length sand packed tubular model 's interfacial tension changes relatively stable when the ternary system injection PV is $0 \sim 6.0 \mathrm{PV}$, and the value is relatively high, all of the ternary was adsorbed on the surface of the oil sands approximately, after the number of injection PV is 6.0, the interfacial tension decreases slowly, the ternary begins to 
flow out, the capacity of adsorption continues to increase, when the number of injection PV is 8.0, the interfacial tension reaches the state of original ultra-low, compared with the natural sand packed tubular model's adsorption experiment without residual oil, with the increase of the amount of injection, the interfacial tension is not stable, but fluctuates within a certain range of ultra-low state, this is due to the oil changes in the produced liquid, which is causes the different content of the surfactant, it can be considered when the injection volume of ternary is 8.0 PV, the amount of adsorption reaches the maximum, the saturated adsorption capacity of this oil sand is $5.13 \mathrm{mg} / \mathrm{g}$ by the calculation. Which indicate that the surfactant front is a non-piston type movement.

There are many factors affecting the adsorption capacity of the binary system and ternary system, the residual oil in the sand packed tubular model is the factor affecting the amount of adsorption. When there is residual oil in the sand packed tubular model, on the one hand, the surfactant gather in the interface of residual oil and water, which consumes some of the surfactant. On the other hand, the oil film on the oil sands separates the surfactant from the oil sands, reducing the contact surface area of the oil sands and surfactant, thus reducing the adsorption of the surfactant on the oil sands surface. By comparing Fig. 1 and Fig. 2, binary system saturated adsorption capacity is $18.92 \mathrm{mg} / \mathrm{g}$ in the sand packed tubular model without residual oil, the adsorption capacity is $6.67 \mathrm{mg} / \mathrm{g}$ in the sand packed tubular model with residual oil, the amount of adsorption capacity is bigger when the sand packed tubular model without residual oil, indicating that the first influencing factors play a leading role in the amount of surfactant adsorption. By comparing Fig. 3 and Fig. 4, ternary system saturated adsorption capacity is $3.52 \mathrm{mg} / \mathrm{g}$ in the sand packed tubular model without residual oil, the adsorption capacity is $5.13 \mathrm{mg} / \mathrm{g}$ in the sand packed tubular model with residual oil, the amount of adsorption capacity is bigger when the sand packed tubular model with residual oil, indicating that the second influencing factors play a leading role in the amount of surfactant adsorption.

\subsection{The binary system and ternary system adsorption law in the natural cores}

In order to compare the natural cores with the sand packed tubular model adsorption law of binary system and ternary system, experiments about the adsorption law of binary system and ternary system of natural cores were made respectively.

3.3.1 The binary system adsorption law in the natural cores

Using the interfacial tension data, making the curve of the produced liquid's interfacial tension of natural cores (length $50 \mathrm{~cm}$ ) and the binary system injection PV, it is shown in Fig.5.

As can be seen from Fig. 5, the produced liquid of $50 \mathrm{~cm}$ in length natural core 's interfacial tension changes relatively stable when the binary system injection PV is $0 \sim 27.4 \mathrm{PV}$, and the value is relatively high, all of the betaine was adsorbed on the surface of the oil sands approximately, after the number of injection PV is 27.4 , the interfacial tension decreases rapidly, the betaine begins to flow out, the capacity of adsorption continues to increase, when the number of injection PV is $28.4 \sim$ 37.3PV, the interfacial tension decreases slowly, when the number of injection PV is 37.3 , the interfacial tension reaches the state of ultra-low, it can be considered when the injection volume of betaine is $37.3 \mathrm{PV}$, the amount of adsorption reaches the maximum, the saturated adsorption of this oil sand is $18.08 \mathrm{mg} / \mathrm{g}$ by the calculation. Which indicate that the surfactant front is a piston type movement.

3.3.2 The ternary system adsorption law in the natural cores

Using the interfacial tension data, making the curve of the produced liquid's interfacial tension of natural cores (length $50 \mathrm{~cm}$ ) and the ternary system injection PV, it is shown in Fig.6.

As can be seen from Fig. 6, The produced liquid of $50 \mathrm{~cm}$ in length natural core 's interfacial tension changes relatively stable when the ternary system injection PV is $0 \sim 5.6 \mathrm{PV}$, and the value is relatively high, all of the ternary was adsorbed on the surface of the oil sands approximately, when the number of injection PV is 5.6, the interfacial tension decreases rapidly, ternary begins to flow out, the capacity of adsorption continues to increase, when the number of injection is $6.0 \mathrm{PV}$, and the interfacial tension reaches the state of original ultra-low, and with increase of the number of injection volume, the interfacial tension is stable and invariant, it can be considered when the injection volume of mahogany sulfonate is $6.0 \mathrm{PV}$, the amount of adsorption reaches the maximum, the saturated adsorption of this oil 
sands is $2.80 \mathrm{mg} / \mathrm{g}$ by the calculation. Which indicate that the surfactant front is a piston type movement.

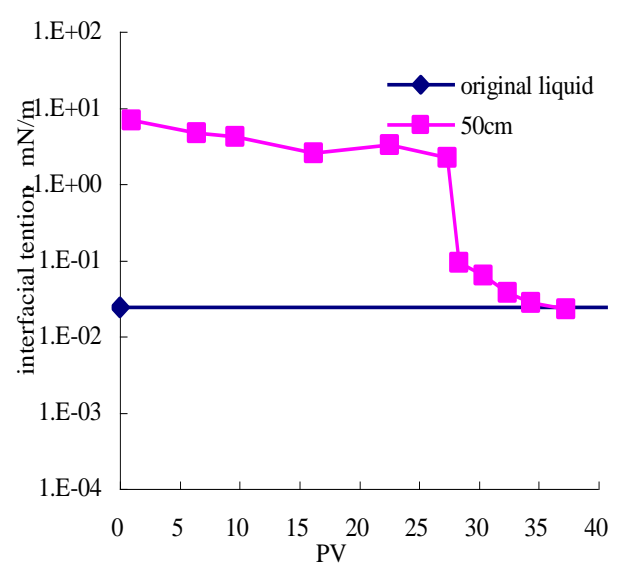

Fig.5 Relation of interfacial tension with PV

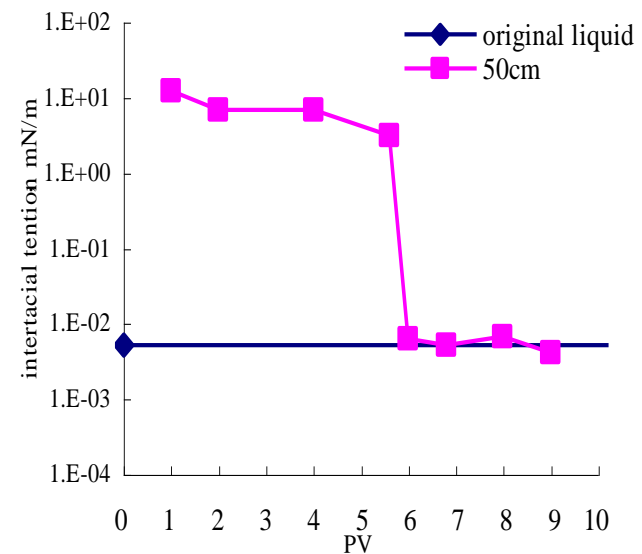

Fig.6 Relation of interfacial tension with PV

(with residual oil)

In the dynamic adsorption experiment of sand packed tubular model with residual oil, when the injection volume of binary system is $10.4 \mathrm{PV}$ and the injection volume of ternary system is $8 \mathrm{PV}$, the interfacial tension of the produced liquid can reach the original ultra-low. After polymer flooding, binary compound flooding and ternary compound flooding can greatly improve the oil recovery. The laboratory experiment showed that: $0.3 \mathrm{PV}$ betaine binary system or $0.3 \mathrm{PV}$ mahogany sulfonate ternary system could increase the recovery rate $10 \%$ above ${ }^{[8]}$. When well spacing is $106 \mathrm{~m}$ in the oilfield test, the cumulative injection ternary system of $0.603 \mathrm{PV}$ can improve the recovery rate $21.4 \%$ than that of water flooding ${ }^{[9]}$. According to this calculation: The reasonable slug $(0.3 \mathrm{PV})$ in laboratory flooding is $2.88 \%$ of the injection volume $(10.4 \mathrm{PV})$ that the interfacial tension reaches original ultra-low. That is, the interface tension of the binary system - crude oil reaches original ultra-low only within the injection of $1.442 \mathrm{~cm}$ in the length $50 \mathrm{~cm}$ Daqing class III sand packed tubular model. The reasonable slug $(0.3$ PV) in laboratory flooding is $3.75 \%$ of the injection volume $(8.0 \mathrm{PV})$ that the interfacial tension reaches original ultra-low, That is, the interface tension of the ternary system - crude oil reaches original ultra-low only within the injection of $1.875 \mathrm{~cm}$ in the length $50 \mathrm{~cm}$ Daqing class III sand packed tubular model.

According to the calculation of five point injection production well pattern, when well spacing is $106 \mathrm{~m}$ in the oilfield test, the cumulative injection ternary system of $0.603 \mathrm{PV}$ can improve the recovery rate $21.4 \%$ than that of water flooding ${ }^{[9]}$, the interfacial tension between the flooding system and crude is reaches ultra-low in the range of $46.45 \mathrm{~m}$ of the injection well's bottom radius (27.35\% of its control area). So it can be concluded: The adsorption losses in the displacement migration process reduces its concentration of effective ingredients, although the interfacial tension of binary or ternary flooding system - crude oil in the reservoir is not all in the original ultra-low, but depend on the emulsification of ultra-low interfacial tension within a radius of injection well and synergy with other components can effectively improve the oil recovery.

\section{Conclusion}

(1) The adsorption migration law of binary system and ternary system in the natural core is consistent with the natural sand packed tubular model, the surfactant front shows the characteristics of piston propulsion mostly.

(2) When the produced liquid reaches the ultra-low interfacial tension, the interfacial tension without residual oil is relatively stable, and the interfacial tension with residual oil fluctuates up and down.

(3) Under this experimental conditions. In the a natural sand packed tubular models without oil and natural cores, the adsorption capacity of betaine binary system is 5.4 and 6.5 times than mahogany 
sulfonate ternary system respectively, and the difference is very large . In the a natural sand packed tubular models with oil, the adsorption capacity of betaine binary system is 1.3 times than mahogany sulfonate ternary system, and the difference is very small.

(4) The interfacial tension of binary or ternary flooding system - crude oil in the reservoir is not all in the original ultra-low, but depend on the emulsification of ultra-low interfacial tension within a radius of injection well and synergy with other components can effectively improve the oil recovery.

\section{Acknowledgements}

The 13th Five-Year national major projects (2017ZX05009004-005)"Feasibility study of composite gas to improve $\mathrm{CO}_{2}$ gas flooding in low permeability-extra low permeability reservoir"

\section{References}

[1]. Daoshan Li, Jirui Hou, Ruijuan Xu. Adsorption of Components from ASP Flooding Solution onto Reservoir Rock of Daqing[J]. Oil Chemistry, 2001(12):358-361.

[2]. Libo Zhang, Hongyan Cai, Qiang Wang et al. Study on static adsorption of each components during the process of ASP flooding[J].Petroleum Geology and Recovery Efficiency, 2014, 21(2):32-34.

[3]. Bailin Li, Yingying Zhang, Sujuan Dai et al. Adsorption properties of ASP flooding system for the central Saertu sub reservoir in Daqing[J].Journal of Northeast Petroleum University, 2014, 38(6):92-99.

[4]. Gang Chen. The Study on the Characteristics and Oil Displacement Effect of Non-alkali Binary Compound System in Sazhong Oilfisld[D]. Northeast Petroluem University.2015.

[5]. Lei Zhang. Study on Characteristics of Adsorption and Retention of Amphiphilic Polymers and Surfactants on Rocks and Minerals [D].China University of Petroleum,2013.

[6]. Huihui Kou, Jingsheng Cui, Hou Dongdong et al. Experimental Study on Multilevel Adsorption of SP Binary System [J].Oil Chemistry, 2013(06):231-234.

[7].Shidong Wu. Study on Static Adsorption of ASP System [J].Chemical Engineer, 2016(06): 34-36+64.

[8]. Junwei Chen. Experimental Study on Application of Betaine System in the Class III Reservoir Daqing[D].Northeast Petroluem University,2017.

[9]. Jiecheng Chen, Guangzhi Liao, Zhenyu Yang et al. Pilot Test of ASP Flooding in Daqing Oilfeild [J].Petroleum Geology Oilfield Development in Daqing.2011 (04):46-49. 